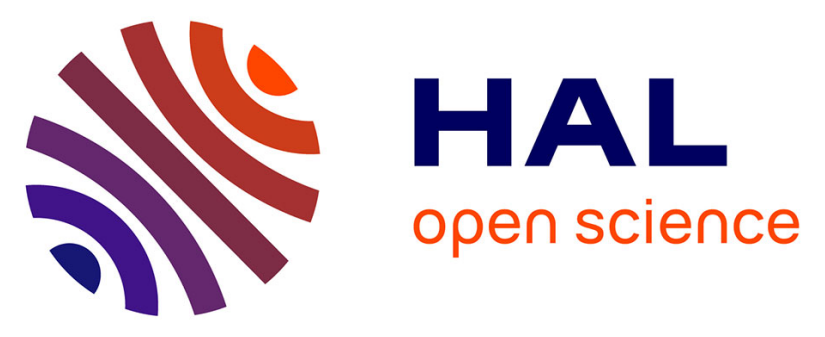

\title{
La fraude est-elle gérable? Application au cas des assurances complémentaires santé
}

\author{
Nicolas Dufour, Emmanuel Laffort
}

\section{To cite this version:}

Nicolas Dufour, Emmanuel Laffort. La fraude est-elle gérable? Application au cas des assurances complémentaires santé. Colloque de Nice / 6-7 décembre 2001 La communication d'entreprise - Regards croisés Sciences de Gestion / Sciences de l'information et de la communication Actes du 5ème colloque du Centre de Recherches en Information et Communication, 2018, 126, pp.211-237. hal-03335959

\section{HAL Id: hal-03335959 \\ https://hal.science/hal-03335959}

Submitted on 6 Sep 2021

HAL is a multi-disciplinary open access archive for the deposit and dissemination of scientific research documents, whether they are published or not. The documents may come from teaching and research institutions in France or abroad, or from public or private research centers.
L'archive ouverte pluridisciplinaire HAL, est destinée au dépôt et à la diffusion de documents scientifiques de niveau recherche, publiés ou non, émanant des établissements d'enseignement et de recherche français ou étrangers, des laboratoires publics ou privés. 


\title{
La fraude est-elle gérable? Application au cas des assurances complémentaires santé
}

\author{
Nicolas Dufour \\ Docteur en gestion, Professeur affilié \\ Paris School of Business \\ (France) \\ Emmanuel Laffort \\ Docteur en gestion, Chercheur associé au CREG, \\ Université de Pau et des Pays de l'Adour
}

(France)

La fraude est un objet d'étude conjoncturel alors qu'elle devrait faire l'objet d'une attention continue et soutenue. Elle est étudiée lorsqu'elle fait parler d'elle puis tombe dans un certain oubli malgré certaines initiatives ponctuelles. Malheureusement, ces initiatives sont souvent normatives et peuvent laisser croire que les organisations sont raisonnablement prémunies dès lors qu'elles suivent les schémas prescrits, ce qui questionne sur la capacité des organisations à structurer la réponse aux fraudes. En nous fondant sur une étude de cas concernant les entreprises proposant des assurances complémentaires santé, nous suggérons que la fraude est également un enjeu d'appropriation.

Mots-clés : Risque opérationnel, fraude, appropriation, Enactment, complémentaire santé

Fraud is a cyclical field of study while it should be a continuous one. The field is studied when fraud comes in the newspaper but despite some isolated initiatives - become more or less neglected as time passes and until next case. Unfortunately, these initiatives are often normative and organizations may feel reasonably protected from fraud in following anti-fraud schemes. This raises the question of the capacity of organizations to adopt a structured response against fraud. Based on a case study in the field of complementary health insurance, we suggest that considering appropriation is a useful way to help managing fraud. 
Keywords: Operational Risk, Fraud, Appropriation, Enactment, Complementary Health Insurance

Blabla

Palabras claves : Riesgo operacional, Blabla

\section{Introduction}

Les cadres théoriques pour penser la fraude ne manquent pas et sont un objet d'étude depuis près d'un siècle avec les travaux séminaux de Sutherland dès 1924 (Sutherland et al., 1992) et l'introduction du terme de criminel en col blanc. Toutefois, les apports de la recherche en gestion dans ce domaine sont encore parcellaires face au caractère évolutif et à la nécessité d'une vigilance constante des organisations face aux fraudes. En outre, la fraude est un enjeu sous-estimé pour la majorité des TPE-PME et l'actualité récente montre que même des entreprises de taille critique ayant mis en place des dispositifs de contrôle et d'audit peuvent être affectées et ce quels que soient les secteurs (Michelin, Intermarché, KPMG, Sony, Goldman Sachs, Malakoff-Médéric pour ne citer que ces exemples).

Les approches possibles en sciences de gestion nous amènent à une question fondamentale consistant à déterminer si la fraude est en soi gérable. Notre question de recherche est la suivante: une organisation peut-elle structurer un processus de lutte anti-fraude efficace et comment? La lutte contre la fraude peut-elle s'envisager comme un processus proactif?

Répondre à une telle question suppose également de prendre en compte le caractère situé de la fraude. À cet effet, nous proposons d'étudier le cas des "fraudes aux complémentaires santé », en tant que cas d'étude sectoriel.

Les pratiques frauduleuses dans le secteur des entreprises proposant des assurances complémentaires santé sont un sujet d'attention récurrent pour les pouvoirs publics (ministère des Finances, Sécurité Sociale), évoquant des montants de fraudes détectées supérieurs à 150 millions d'euros/an en tendanciel. Une telle somme néglige les fraudes subies par les organismes distributeurs et gestionnaires des complémentaires santé (mutuelles). L'actualité récente montre l'intérêt d'un tel sujet et la nécessité pour ces organismes de mettre en œuvre de véritables dispositifs de lutte antifraude face à des pratiques de fraudes s'étant professionnalisées, mais 
aussi ayant connu un développement exponentiel, constituant ainsi une tendance lourde ${ }^{1}$. L'étude des fraudes par ce spectre sectoriel semble adaptée pour renforcer la compréhension d'un besoin en termes de réponse gestionnaire face à ce risque opérationnel. Après avoir présenté les fondements académiques et conceptuels structurants, nous développons une étude de cas menée sur une longue durée en recherche-action. Nous envisageons ensuite les apports théoriques et managériaux issus de notre étude.

\section{La fraude, naissance formelle et combat ordinaire}

\subsection{Une prise en compte formelle récente}

La fraude est un objet de recherche récent, qui a été peu étudié jusque dans les années 1990 (Ouimet, 2012) et qui doit beaucoup aux travaux de Sutherland (1940, 1944 ; Sutherland et al., 1934) et de Cressey (1950, 1955, 1986). Sutherland, l'inventeur du terme " Criminel à col blanc », a refusé de séparer la criminalité en col blanc de la délinquance issue des milieux défavorisés et a proposé une théorie explicative, l'association différentielle. Cressey a poursuivi dans cette voie et en approfondit l'explication sociologique. Selon lui, le fraudeur est engagé dans son action délictueuse sous l'effet principal de trois dimensions: la pression subie, la motivation à frauder et la rationalisation de l'acte, il s'agit du fameux « triangle de la fraude ». Cette voie continue d'être approfondie aujourd'hui, et on trouve des travaux récents mobilisant ce triangle, y compris dans la norme ( I.F.A.C., 2009) ou tentant de le compléter, parfois à l'aide d'autres figures géométriques (Gbegi, Adebisi, 2013; Kassem, Higson, 2012; Lanier, 2010; Mackevicius, Giriunas, 2013) mais toujours à l'aide de dimensions sociologiques.

Ces réflexions ont permis des avancées significatives conduisant en particulier à reconnaître une nouvelle catégorie de risques: le risque opérationnel ${ }^{2}$, dont le risque de fraude. Les régulateurs et les normalisateurs de différents pays se sont également saisis du sujet

\footnotetext{
${ }^{1}$ Voir les études UFC-Que Choisir (2014), EY (2014), PWC (2015) ainsi que le baromètre du Risk Manager 2015 (AMRAE) soulignant le caractère prioritaire de la fraude comme enjeu de gestion des risques.

${ }^{2}$ Dans le secteur bancaire et financier, par exemple, le Comité de Bâle s'est saisi du sujet en 1998 pour arriver à une acception en partie partagée de ce risque en 2003.
} 
pour prendre en compte ce risque, et la prise de conscience fut assez rapide, en particulier dans des domaines où les professionnels avaient l'habitude d'être confrontés au problème. C'est le cas pour les normes d'audit des comptes avec la norme ISA 240 (I.F.A.C., 2009) relative à la responsabilité de l'auditeur dans le cadre de l'audit financier établi par la fédération internationale des experts comptables. Cette norme, par ailleurs très complète, se concentre sur la fraude aux états financiers (IAS 240). Elle distingue l'erreur dans les états financiers de la fraude par son caractère intentionnel et définit la fraude comme l'emploi d'une manœuvre trompeuse dans le but d'obtenir un avantage indu ou illégal. C'est d'ailleurs un caractère que l'on retrouve dans les différentes normes traitant de ce sujet tant aux États-Unis qu'au Canada (CICIA, chap. 5135) et en France (CNCC, NEP-240; Smaili et al., 2009). Il semble d'ailleurs que cette acception de fraude soit celle qui retienne principalement l'attention des praticiens. Ainsi, Ouaniche (2015) se concentre-t-il également sur la fraude comptable et les normes afférentes.

On peut noter que malgré cette prise de conscience de la matérialité de la fraude, elle reste encore le parent pauvre du risque opérationnel, au moins en ce qui concerne l'autorité de tutelle des mutuelles de santé.

Le $\mathrm{COSO}^{3}$, une organisation indépendante issue de cinq organisations du secteur privé, dont le but est de développer des cadres et de proposer des recommandations concernant la gestion du risque des entreprises et du contrôle interne devant permettre de dissuader la fraude propose 17 principes pour parvenir à gérer ce risque dont le huitième est dédié à la fraude. Ce cadre propose une acception plus large de la fraude en ce que le principe propose de considérer différents types de fraudes, les facteurs aggravants, les attitudes comportementales (COSO, 2013).

C'est la raison pour laquelle, plutôt que de nous restreindre à un cadre peu adapté aux contrôles qualitatifs et plus aisément contournable (puisque connu), nous préférons élargir le périmètre aux actions malveillantes, y compris futures, issues de schémas encore inconnus ou ignorés (Cressey, 1986).

\subsection{Des repères à construire pour la fraude à l'assurance}

\footnotetext{
${ }^{3}$ Committee of Sponsoring Organizations of the Tradeway Commissions
} 
Dès le début, la fraude a du faire face à un problème de définition, pour (Cressey, 1950), la fraude n'est pas définie, mais peut être caractérisée. Nous retrouvons aujourd'hui la faiblesse des définitions : alors que de nombreux documents émanant des régulateurs et des normalisateurs recommandent de porter une plus grande attention à la fraude, elle n'est nulle part définie, mais souvent citée parmi d'autres actions répréhensibles : "[...] any improper or illegal activity, such as money laundering, fraud, bribery or corruption." (B.C.B.S., 2010, p. 9). D'ailleurs ce n'est pas forcément un problème puisque nous savons tous bien ce que représente la fraude... Mais peut-être est-ce la première question à se poser, lorsque « tout le monde sait» sommesnous autre part que dans le présupposé (Deleuze, 2014) ou comme nous le rappelle Jullien (2012) « Ce qui est "bien connu", disait Hegel, n'est, de ce fait, pas connu $\gg .$.

Dans le secteur des assurances, seuls de rares articles tendent à proposer une définition de ce qu'est la fraude et encore plus rarement un cadre de gestion approprié. Citons l'étude récente de Flynn (2016) proposant un renforcement des technologies et moyens de lutte contre ces fraudes, de même qu'un besoin d'interactions renforcées entre organismes d'assurance privés et d'entreprises fournissant les services de santé. Cette étude n'offre cependant pas de définition précise ni de cadre global transposable aux fraudes aux prestations santé. Il faut alors remonter aux travaux de (Dixon, 1995, 1997) pour envisager une conception de la fraude. Soulignant le coût élevé des fraudes et le fait que les fraudes sont aussi variées que les formes d'organisations criminelles qui les encouragent, l'auteur précise que les fraudes à l'assurance ont très majoritairement comme fondement la production de fausses informations voire de faux justificatifs d'information devant permettre le paiement d'une indemnisation par l'assureur. De telles tendances sont alors expliquées par un contexte pro cyclique - la fréquence des fraudes augmente en période de crise (Blanqué, 2003) et ont structurellement un lien avec la notion d'aléa moral, chaque portefeuille d'assuré implique une part de fraudeur (Okura, 2013). La fraude est donc structurelle (elle est toujours potentielle) et conjoncturelle (sa gravité et sa fréquence dépendant d'un contexte particulier). Sans fournir là encore d'éléments de description de processus anti-fraude, ces éléments permettent d'envisager des actions possibles de réduction des fraudes, comme l'apport des réglementations dissuasives (Swaby, 2011), la communication pour sensibiliser (Tseng, Su, 2013) ou encore l'investissement dans des technologies et des moyens préventifs de détection à l'instar du datamining (Rejesus et al., 2005). 
Il n'en reste pas moins qu'une véritable gestion de la fraude dans le cadre de processus dédiés implique de construire ces repères, $a$ fortiori dans des secteurs encore peu étudiés sur le plan académique, comme le secteur des assurances. Ce constat fait émerger le besoin d'une grille de lecture complémentaire aux autres approches gestionnaires de la fraude.

\subsection{Le recours à l'appropriation. Grille de lecture théorique mobilisée}

La naissance de la notion d'appropriation est diffuse. On la trouve chez (Illich, 1971, p. 216) qui voit dans la technologie un outillage qui permettrait de "s'associer pour accomplir une tâche ou, comme tout bricoleur le sait d'instinct, de découvrir en se servant de nouvelles possibilités d'utilisation ». Ce bricolage, accessible à « [...] l'homme ordinaire. Héros commun. Personnage disséminé » (de Certeau, 1991) lui permettant de créer «[...] un espace de jeu pour des manières d'utiliser l'ordre contraignant du lieu ou de la langue». L'appropriation consiste bien à inventer son quotidien, c'est un processus complexe, un «art», dont de Vaujany (2006) propose une grille de lecture selon trois perspectives conjointes. Ainsi mobilisée, l'appropriation permet de concevoir l'action collective (Dechamp et al., 2006).

Ces trois perspectives (rationnelle, socio-politique et psychocognitive) permettent de " se mettre à la place " de tous les types d'acteurs concernés par le sujet en question et d'en comprendre les aspirations, plus ou moins conscientes, les structures dans lesquelles ils sont placés, les enjeux auxquels ils sont soumis et dans lesquels ils sont parties prenantes.

- Dans la perspective rationnelle, le point de vue que l'on cherche à capter est celui d'acteurs ayant une vision normative de leur environnement. Mais cette vision n'est pas uniquement à placer dans une perspective " en toute rationalité ", elle dépend des processus personnels soumis au poids des représentations sociales.

- Dans la perspective socio-politique, on va chercher à comprendre la façon dont les acteurs s'inscrivent au sein de l'organisation et comment ils imaginent instrumentaliser leur environnement afin de conforter ou d'améliorer cette inscription. Il s'agit de tenter de comprendre les dynamiques individuelles (inscrites dans un contexte social), les dynamiques sociales et d'anticiper l'incidence de l'appropriation de leur environnement sur ces dynamiques. 
- Enfin, dans la perspective psycho-cognitive, on cherche à comprendre comment les acteurs construisent leurs réalités et de quelles façons leur environnement vient modifier les schèmes cognitifs. Il s'agit aussi bien d'être attentif aux objets symboliques (en particulier les mythes) qu'aux investissements personnels affectifs ou aux biais cognitifs et de la façon dont tout ceci affecte les rationalités personnelles.

Ces trois perspectives se recouvrent d'ailleurs sur certains points : la perspective rationnelle ne peut pas se comprendre sans un éclairage à la fois psycho-cognitif et socio-politique (il n'existe pas de «toute rationalité » et les acteurs sont toujours «situés»), la perspective socio-politique ne peut pas s'exonérer d'un éclairage psycho-cognitif et réciproquement : comment pouvoir comprendre les interactions sociales si on n'investit pas un minimum les schèmes cognitifs et inversement, comment pouvoir imaginer que ces schèmes sont indépendants de l'incrustation sociale?

Cette notion d'appropriation est très proche de celle d'enactment (Weick, 2001), à telle point qu'elle peut lui être confondue (Hussenot, 2006). Or, selon Weick, le plus important dans cette perspective d'enactment est que cela peut servir de base à une idéologie de prévention et de gestion des crises en permettant la création de sens. C'est en particulier parce que les individus peuvent donner du sens qu'ils peuvent être vigilants et prévenir les problèmes.

L'appropriation est donc une démarche pouvant permettre de comprendre comment un dispositif au sens large est compris, intégré et utilisé par des acteurs.

\section{2 Étude empirique des fraudes aux complémentaires santé, l'apport de la recherche-action}

\subsection{Protocole de la recherche}

Le protocole de recherche utilisé consiste en la réalisation d'une étude de cas longitudinale menée par recours à une démarche méthodologique de type recherche-action. Nous avons réalisé cette étude de cas en tant que Risk Manager, (260 jours-homme sur deux ans) étant partie prenante et ayant un rôle actif dans la construction du dispositif de gestion étudié. La lettre de mission sur cette intervention avait pour objectif la mise en place d'un processus de lutte anti-fraude sur les processus étant au cœur du métier de la mutuelle étudiée (fraudes aux assurances santé et prévoyance). 
2.1.1 Justification du recours à une démarche enracinée et validité de la démarche de recherche

La recherche-action nous semblait justifiée pour plusieurs raisons : au regard de notre objet d'étude (la fraude), un positionnement en tant qu'acteur interne de l'organisation permettait un accès facilité au réel, s'agissant de dispositifs confidentiels et pour lesquels un acteur externe, y compris en mission de consulting, n'aurait eu qu'un accès limité. Il aurait par exemple eu un accès restreint aux cas et schémas de fraude réduisant la possibilité de compréhension du processus étudié.

En outre, une telle méthodologie s'oppose « aux théories produites par déduction logique d'hypothèses définies a priori » (Glaser, Strauss, 2012) et permet de comprendre comment les pratiques sociales et à visée d'apprentissage sont localisées et émergent dans des circonstances empiriques (Kemmis et McTaggart, 2005). La recherche-action nous semble particulièrement adaptée eu égard au caractère exploratoire de notre recherche, s'agissant d'une «méthodologie expérimentale en vue de l'action» (Barbier, 1996) et pour laquelle l'apport d'enseignements managériaux implique de respecter une recherche de contingence générique, ce qui suppose d'être acteur du processus étudié pour discerner entre les nombreuses variables à prendre en compte (Coghlan, Brannick, 2005 ; Savall, Zardet, Boje, 2004).

Enfin, dans une logique de triangulation méthodologique (Jick, 1979 ; Paul, 1996) le recours à cette recherche-action nous a permis de collecter des données primaires complémentaires (entretiens internes et externes) à l'organisation étudiée et des données secondaires issues de nombreux éléments de documentation interne. Les entretiens réalisés nous ont permis de renforcer la validité de nos données et de limiter les biais d'ancrage propre à une étude enracinée ${ }^{4}$.

\section{2 Étude de cas : la mise en place d'un processus de lutte anti- fraude au sein d'une mutuelle}

\subsubsection{Contexte de l'étude}

\footnotetext{
${ }^{4}$ Une limite cependant identifiée tient à la confidentialité de certains résultats de l'étude, sans pour autant dénaturer la restitution faite dans le présent travail. Les données non diffusées sont: nombre et importance des cas de fraudes; éléments confidentiels du processus fraude mis en place.
} 
Les éléments de contexte associés à notre étude, en phase de conception puis de déploiement du processus de lutte anti-fraude, sont les suivants: un processus historiquement inexistant (seuls des contrôles de cohérence entre les opérations réalisées et leurs justificatifs étaient faits) ; certains cas de fraude détectés par hasard permettaient de se rendre compte de la nécessité d'industrialiser la démarche de gestion de la fraude ; l'expertise métier pour la détection des fraudes et le traitement des cas était présente dans différents services, mais cette activité n'était pas coordonnée ni pilotée. Un autre élément de contexte concernait les pertes issues des fraudes avérées : lesquelles justifiaient, par leur montant, d'investir dans un dispositif structuré. Enfin, le cadre prudentiel « Solvabilité II », applicable aux organismes d'assurance et mutuelles à compter du 1er janvier $2016^{5}$, renforce les exigences relatives à la mise en œuvre d'un dispositif de contrôle des risques (Dufour, 2015). Cela implique le renforcement des processus, procédures et actions de contrôles mis en place.

Notre étude prend place au sein d'une mutuelle proposant principalement des contrats de complémentaire santé (frais de soin en optique, dentaire, hospitalisation, kinésithérapie, services d'assistance ambulances, services d'infirmières à domicile, etc.), ce auprès de plus de 300000 particuliers et professionnels sur l'ensemble du territoire français. La structure étudiée, de taille moyenne, est une PME de moins de 500 collaborateurs. Le contexte de la mutuelle au début de l'étude est celui d'une prise de conscience du Conseil d'Administration et de la Direction Générale de la nécessité de gérer le risque de fraude pour répondre à un double enjeu : de renforcement du dispositif de contrôle interne sur son cœur de métier dans le cadre de la directive Solvabilité II et de maîtrise du coût des prestations payées aux adhérents, a fortiori sur les cas d'abus et de fraude, tenant compte de différentes études sur la fraude.

2.2.2 Design du processus de lutte anti-fraude : fournir le terreau favorable à l'appropriation

La question de recherche évoquée en introduction (une organisation peut-elle structurer un processus efficace de lutte antifraude et comment?) émane d'une préoccupation pratique de la Direction Générale: "La fraude c'est un risque subi, il y en a toujours eu, et on voit bien qu'aucun acteur n'est réellement en

${ }^{5}$ Directive européenne 2009/138/CE, transposée par l'ordonnance du 2 avril 2015 et le décret du 10 mai 2015. 
avance sur le sujet, même les assureurs ou mutuelles de taille critique maîtrisent peu la fraude aux prestations santé 》 (Direction Générale). De tels propos sont étayés par les opérationnels confrontés au sujet au quotidien: "On ne fait que constater des pertes, il nous faudrait de solides moyens et surtout une organisation claire pour enrayer le phénomène au moins partiellement » (Responsable Gestion).

Face à cette préoccupation, notre étude sur deux ans a consisté à concevoir et mettre en œuvre un processus de gestion de la fraude.

La première étape a consisté à définir la lutte anti-fraude comme un enjeu prioritaire. Nous nous sommes appuyés sur la cartographie des risques majeurs de l'entreprise pour mettre en évidence que le risque de fraude externe est un risque prioritaire pour la mutuelle. Cela a été fait en tenant compte de la fréquence des fraudes - étant entendu que la mutuelle n'avait qu'une faible capacité à détecter les fraudes en début d'étude - de leur impact en cas de survenance (les fraudes dépassent le millier d'euros) et en tenant compte de l'existant en matière de maîtrise des risques (contrôles de cohérence sur justificatifs).

En second lieu, plusieurs stratégies de lutte anti-fraude ont été définies. Sur les fraudes de faible montant, une stratégie d'acceptation du risque en l'état a été définie, afin de concentrer les ressources limitées sur les cas les plus coûteux ou les plus impactant en termes d'image pour la mutuelle. Sur certains schémas de fraude (comme sur les frais dentaires), une stratégie de dissuasion (réponse civile et pénale) a été définie indépendamment du coût de la lutte anti-fraude. Une stratégie orientée conformité a été mise en œuvre pour les cas pouvant être associés à du blanchiment de capitaux. Enfin, pour les cas les plus coûteux une stratégie orientée recouvrement des sommes détournées a été définie (amiable, précontentieux ${ }^{6}$ puis contentieux ${ }^{7}$ ). "Avoir une vision claire de ce que l'on voulait faire pour lutter contre la fraude a permis de mieux voir comment on allait répondre à l'enjeu selon les cas. Il nous fallait une stratégie à décliner en pratique. " (Directeur des opérations).

Une fois cette stratégie définie, un groupe de travail a ensuite été mis en place afin de décrire un processus de lutte anti-fraude.

\footnotetext{
${ }^{6}$ Recours à des sociétés de recouvrement ou à des huissiers pour les cas de fraudes avérées.

${ }^{7}$ Action civile ou action civile et pénale à l'encontre des fraudeurs.
} 
L'organisation préexistante se limitant aux contrôles opérationnels, la démarche de mise en place du processus n'impliquait pas une revue d'un processus existant, il s'agissait d'une réorganisation par rupture consistant à mettre en œuvre le processus cible directement. La définition du processus s'est toutefois étalée sur une période de 4 mois, afin de capitaliser sur les différents cas de fraude et de tenir compte des acteurs concernés. Le processus a été conçu dans une logique partenariale et fait appel aux différentes expertises internes sur le sujet avec des professionnels de santé, des experts par type de prestations, des experts de l'organisation, du contrôle, des juristes.

2.2.3 Mise en œuvre du processus : l'appropriation comme socle fondateur

Pour comprendre la façon dont ce processus était approprié, nous l'avons déclinée selon les trois regards de l'appropriation.

Tableau 1. Processus anti-fraude et appropriation

\begin{tabular}{|c|c|}
\hline \multicolumn{2}{|r|}{ Étapes du processus de lutte anti-fraude } \\
\hline \multirow{3}{*}{ 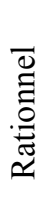 } & Description et revue des enjeux et des obligations en la matière \\
\hline & $\begin{array}{l}\text { Mise à jour de la cartographie des risques (réévaluation des } \\
\text { risques) }\end{array}$ \\
\hline & $\begin{array}{l}\text { Mise en place de tableau de pilotage du processus / de tableau } \\
\text { de suivi }\end{array}$ \\
\hline \multirow{3}{*}{ 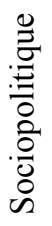 } & $\begin{array}{l}\text { Description et ajustement des actions (qui-fait-quoi, revue de } \\
\text { processus) }\end{array}$ \\
\hline & Actions de communications sur la prévention / dissuasion \\
\hline & $\begin{array}{l}\text { Mise à jour des contrôles. Partage des résultats ; remontées } \\
\text { d'alertes, ... }\end{array}$ \\
\hline \multirow{4}{*}{ 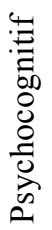 } & Mise à jour du référentiel. Partage sur les cas à anticiper \\
\hline & Partage sur les pratiques constatées ou à promouvoir \\
\hline & $\begin{array}{l}\text { Création d'une base d'incidents: historiques, retours } \\
\text { d'expériences... }\end{array}$ \\
\hline & Retours réguliers sur les raisons et les résultats du processus \\
\hline
\end{tabular}

La déclinaison des processus s'est opérée via la mise en œuvre des différentes actions décrites dans le tableau ci-dessus. Afin de capitaliser sur le dispositif construit, la démarche de recherche-action a consisté à mettre en place une revue annuelle du processus ( 2 revues 
réalisées lors de l'étude) qui consiste à évaluer la performance du processus mis en œuvre ainsi qu'à en réaffirmer l'importance.

L'évaluation de cette performance est envisagée sur un axe quantitatif à l'aide de plusieurs indicateurs tels l'augmentation du nombre de cas détectés; part des cas détectés par rapport aux cas déjoués, temps consacrés à la détection des cas avérés par rapport aux cas non concluants, part des sommes recouvrés sur l'ensemble des coûts des fraudes, retour sur investissement des moyens mis en œuvre pour identifier et traiter les fraudes.

Un axe complémentaire a émergé lors des revues de processus avec les métiers (opérationnels concernés, managers, professionnels de santé agissant en tant qu'experts-conseils pour la mutuelle). Cet axe est fondé sur la démarche d'appropriation mise en œuvre et sur le constat qu'une stratégie de lutte anti-fraude doit prendre en compte trois critères : la responsabilité des acteurs; leur autonomie ; et les sanctions qu'ils encourent (approche RAS). En effet, une réelle appropriation $\mathrm{du}$ processus conduirait les acteurs à plus de responsabilité, ils échangeraient plus, puisqu'ils seront plus conscients des enjeux, et mieux parce qu'ils savent mieux sur quoi communiquer. L'appropriation aura un effet qui peut être pervers : comprenant mieux le système et les contrôles réalisés, ils pourraient le détourner en connaissance de cause, ce qui serait facilité par les moyens de leur action. Le risque est donc d'autant plus important que leur autonomie est forte. Une réponse supplémentaire réside dans la sanction, qui est appelée par les professionnels eux-mêmes. De plus en plus conscients des enjeux, ils souhaitent des sanctions à même de dissuader la fraude et s'engagent avec détermination dans ce processus.

Tableau 2. Processus anti-fraude et démarche RAS

\begin{tabular}{|c|c|}
$\begin{array}{c}\text { Approche } \\
\text { RAS }\end{array}$ & $\begin{array}{c}\text { Rôle de l'appropriation dans le renforcement de ces } \\
\text { axes (verbatim et démarche) }\end{array}$ \\
\hline
\end{tabular}




\begin{tabular}{|c|c|}
\hline \multirow{4}{*}{$\begin{array}{l}\text { Capacité / } \\
\text { incitation des } \\
\text { acteurs } \\
\text { internes ou } \\
\text { externes à } \\
\text { l'organisation } \\
\text { à rendre des } \\
\text { comptes }\end{array}$} & $\begin{array}{l}\text { «Echanger avec les dentistes-conseils, opticiens- } \\
\text { conseils ou médecin-conseils nous permet de voir ce qui } \\
\text { sort de la normale, non seulement en termes de } \\
\text { montants, mais aussi en termes de pratiques abusives } \\
\text { voire frauduleuses sur les remboursements, sur les } \\
\text { dispositifs médicaux facturés, etc. On sait sur quel sujet } \\
\text { les professionnels de santé et les opérationnels doivent } \\
\text { rendre des comptes et documenter» (Contrôleur des } \\
\text { opérations) }\end{array}$ \\
\hline & $\begin{array}{l}\text { Organisation : La formalisation de processus en } \\
\text { interaction entre contrôleurs, juristes, opérationnels et } \\
\text { experts techniques (optiques, dentaires, hospitaliers, } \\
\text { ambulanciers) }\end{array}$ \\
\hline & $\begin{array}{l}\text { Contrôles: automatisation des rapprochements entre } \\
\text { éléments de preuve et repérage statistiques des cas hors } \\
\text { loi normale }+ \text { investigation détaillée ; demandes de } \\
\text { remontées de reportings réguliers }\end{array}$ \\
\hline & $\begin{array}{l}\text { Pilotage: intégration d'indicateurs de suivi des fraudes } \\
\text { et fixation de limites de risques opérationnels (politique } \\
\text { de maîtrise des risques) }\end{array}$ \\
\hline \multirow{2}{*}{$\begin{array}{l}\text { A } \\
\text { Marge de } \\
\text { manœuvre } \\
\text { qu'ont les } \\
\text { acteurs dans la } \\
\text { pratique } \\
\text { (paiement de } \\
\text { prestations) }\end{array}$} & $\begin{array}{l}\text { «Plus un professionnel de santé a d'autonomie dans la } \\
\text { facturation, plus il est incité à frauder, on le voit avec le } \\
\text { tiers-payant, cela incite un opticien à frauder, surtout } \\
\text { dans un contexte où les mutuelles resserrent les } \\
\text { remboursements. Sans l'aide des experts des professions } \\
\text { de santé, on ne pourrait pas construire de contrôles } \\
\text { adaptés sur les vraies pratiques frauduleuses " } \\
\text { (Responsable Gestion) }\end{array}$ \\
\hline & $\begin{array}{l}\text { Prévention / sensibilisation : traçage des profils et } \\
\text { schémas récurrents ou probables ; mise en place de } \\
\text { points clés de suspicion (Rex et signaux faibles); } \\
\text { communication auprès des adhérents et professionnels } \\
\text { de santé (bulletins et magazine adhérents, site internet). }\end{array}$ \\
\hline
\end{tabular}




\begin{tabular}{|c|l|}
\hline$S$ & $\begin{array}{l}\text { «Si l'on ne sanctionne pas les fraudeurs, cela déteint sur } \\
\text { l'ensemble de la profession, alors contribuer à aider les } \\
\text { assureurs permet de reconnaitre le travail des } \\
\text { professionnels de santé ainsi que le rôle des adhérents }\end{array}$ \\
responsables, qu'on a tendance à oublier dans le débat \\
Capacité de \\
l'organisation \\
à réagir et \\
sanctionner les l'explosion des dépenses de santé » (Opticien- \\
cas de fraude & $\begin{array}{l}\text { Traitement financier : recouvrement des sommes } \\
\text { fraudées (amiables, contentieux civil et pénal), blocages } \\
\text { des cas de suspicions. Diffusion des cas de fraude dans } \\
\text { des bulletins spécialisés à destination des professionnels. }\end{array}$ \\
\hline
\end{tabular}

L'appropriation, en permettant d'identifier une communauté d'acteurs de professions différentes permet un partage de connaissances et de pratiques qui renforce les axes de vigilance en permettant d'améliorer les axes de l'approche RAS : où positionner les contrôles et les reportings à des fins de responsabilisation? Où l'autonomie des acteurs peut-elle être source de fraude? Dans quelle mesure les sanctions, qu'elles soient ou non mises en œuvre, ont-elles un rôle dissuasif?

\section{Discussion des résultats: vers un modèle d'intention théorique}

\subsection{Apport managérial : les fraudes dans le secteur santé, le visage sous-estimé du risque opérationnel}

Surtout lorsque les acteurs sont disséminés au sein d'un processus complexe, l'appropriation est un enjeu managérial. Pour ne pas tomber dans le piège du présupposé dénoncé par Hegel et Deleuze, il est nécessaire de régulièrement vérifier et d'encourager les appropriations des parties prenantes. Cela suppose des conditions nécessaires qui aujourd'hui sont un environnement de contrôle (appui de la gouvernance ; politique dédiée...) ; une organisation mise en place dans cette logique; une atmosphère créant une dynamique autour de l'enjeu de gestion de la fraude (qui est un sujet fédérateur en risk management). Il s'agit donc d'en faire un objectif prioritaire au regard des axes de responsabilisation des acteurs, de maitrise de l'autonomie des activités externes et internes à l'entreprise et de sanction des comportements frauduleux (Frigo, Andersen, 2011). 
Cette démarche fait émerger différents axes de renforcement de la lutte contre la fraude, autour des axes de responsabilisation (des équipes, des adhérents, des professionnels de santé), d'autonomie (des équipes, des professionnels de santé et partenaires), et de sanction (des adhérents, des professionnels de santé, voire des collaborateurs en cas de fraude interne).

Une limite pratique de notre étude reste cependant la maîtrise de l'effet de report en termes de fraude et la capacité d'adaptation des fraudeurs (lorsqu'un schéma de fraude est connu, les fraudeurs «professionnels » chercheront d'autres moyens minimisant le risque d'être pris). Si tel n'est pas l'objet de l'étude, on remarque cependant que la démarche d'appropriation et le partage réguliers d'expériences avec les experts métiers concernés permet justement de concevoir suffisamment tôt les contrôles nécessaires pour détecter voire déjouer des cas de fraude.

\subsection{Apport théorique : la fraude entre responsabilité, autonomie et sanction}

Notre étude se situe dans le prolongement de recherches récentes en contrôle des risques notamment, car elle complète et précise le cadre de pensée sur le risque opérationnel (Hoffman, 2002 ; Jebrin et Abu-Salma, 2012 ; Torre-Enciso et Barros, 2013); ainsi que sur les enjeux éthiques associés à la perspective gestionnaire de risque (Cherré, Dufour, 2015; Meric, Pesqueux, et Sole, 2009). Le renforcement de la connaissance sur la construction d'un processus de lutte anti-fraude constitue en soi un complément à la demande d'une opérationnalisation des démarches de contrôle des risques (Mikes, 2007). Son apport est de contribuer à formaliser des résultats sur un secteur encore peu étudié : le secteur des assurances et plus spécifiquement celui des complémentaires santé, pour lequel les études sont peu nombreuses et datent des travaux de Dixon dans les années 1990 (Okura, 2013 ; Flynn, 2016).

Ces résultats appellent également un approfondissement de la démarche d'appropriation. En particulier, nous souhaitons voir les effets de la démarche RAS sur la qualité de la lutte contre la fraude et nous comptons mobiliser sur ce terrain une démarche d'appropriation croisée qui vise à mobiliser les trois regards de l'appropriation de façon croisée non sur Ego (soi-même), mais sur un partenaire professionnel (Alter), qui, lui-même, la mobilisera de la même façon sur Ego. Elle conduit à une meilleure connaissance de l'activité d'Alter afin de mettre en perspective le métier d'Ego et de le 
décloisonner. La démarche se décompose en deux parties, la première conduisant à l'élaboration des Facteurs Critiques Perçus (FCP) alors que la seconde, itérative, met en œuvre un outil, la balance appropriative, qui permet d'évaluer et d'améliorer la qualité de la relation (Laffort, Cargnello-Charles, 2013).

Cette étude, encore à mi-chemin, monter l'importance de l'appropriation en tant que grille conceptuelle renforçant la théorie gestionnaire du risque (Meric et al., 2009 ; Mikes, 2007; Pathak, Pathak, 2005 ; Power, 2009) et incite à approfondir l'appropriation corisée. Elle s'inscrit également en complémentarité des travaux sur les manières de caractériser les incitations à frauder une activité donnée (Cressey, 1955 ; Kassem, Higson, 2012).

\section{Conclusion}

Les conclusions de cette étude exploratoire sont riches de perspectives et appellent des approfondissements dans le cadre d'une recherche confirmatoire. Ces premiers résultats confirment la nécessité d'envisager la fraude via des grilles de lecture renouvelées et complémentaires aux modèles déjà établis. Nous préconisons des approches fondées sur l'appropriation mettant en jeu des éléments subjectifs personnels et groupaux et qu'il nous semble à approfondir en mettant en œuvre une démarche d'appropriation croisée. Cette approche permet la mise en place d'un processus transverse, entre acteurs du contrôle et du Risk Management, juristes, direction de l'entreprise, opérationnels et experts des activités susceptibles de subir des fraudes. Nous insistons également sur l'apport d'un modèle d'intention théorique fondé sur un triptyque de responsabilité, d'autonomie et de sanction, complémentaire aux travaux de Cressey (1950) notamment que nous comptons évaluer à l'aide de la balance appropriative. Les résultats ont enfin le mérite de proposer une démarche effective de lutte anti-fraude dans le secteur des mutuelles santé, pour lesquelles cette lutte est une véritable préoccupation du fait notamment des contraintes normatives Solvabilité II contrats solidaires et responsables.

\section{REFERENCES BIBLIOGRAPHIQUES}

B.C.B.S. « Gestion du risque opérationnel », 1998, 
B.C.B.S. " Saines pratiques pour la gestion et la surveillance du risque opérationnel », 2003

B.C.B.S. «Principles for enhancing corporate governance - For comment », 2010

BARBIER, R. La recherche action, Anthropos diff. Economica, Paris, 1996.

BlanquÉ, P., "Crisis and fraud », Journal of Financial Regulation and Compliance, Vol. 11, $\mathrm{N}^{\circ}$ 1, 2003, p.60-70.

Cherré, B. et Dufour, N., " Le contrôle en dualité, entre aliénation et autonomie. Le cas du management éthique appliqué aux risques opérationnels », Recherches en Sciences de Gestion, N 108, 2015.

COGHLAN, D. ET BRANNICK, T. Doing action research in your own organization, Sage Publications, London; Thousand Oaks, 2005.

COSO. Enterprise Risk Management for banks; 2013

Cressey, D. R., "The Criminal Violation of Financial Trust ", American Sociological Review, Vol. 15, N ${ }^{\circ} 6,1950$, p.738-743.

CRessey, D. R., " Changing Criminals: The Application of the Theory of Differential Association », American Journal of Sociology, Vol. 61, $\mathrm{N}^{\circ} 2,1955$, p.116-120.

CRessey, D. R., " Why managers commit fraud », AUST \& NZ Journal of Criminology, Vol. 19, 1986, p.195-209.

De Certeau, M. L'invention du Quotidien. I. Les arts de faire (Fiche de lecture), Folio, 1991 [1980].

de Vaujany, F.-X., « Pour une théorie de l'appropriation des outils de gestion : vers un dépassement de l'opposition conception-usage ", Revue Management et Avenir, Vol. 3, $\mathrm{N}^{\circ}$ 9, 2006, p.109-126.

Dechamp, G., Goy, H., Grimand, A. et De Vaujany, F.-X., « Management stratégique et dynamiques d'appropriation des outils de gestion : proposition d'une grille de lecture ", Revue Management et Avenir, Vol. 3, $\mathrm{N}^{\circ}$ 9, 2006, p.181-200.

Deleuze, G. Différence et répétition, Presses universitaires de France, Paris, 2014 [1968].

DiXon, M. I., « Insurance Fraud », Journal of Financial Crime, Vol. 3, № 2, 1995, p.168-169.

Dixon, M. I., « Recent Initiatives in the Prevention and Detection of Insurance Fraud », Journal of Financial Crime, Vol. 4, N 3, 1997, p.236241.

Dufour, N., « L'articulation des fonctions clés sous Solvabilité II: le nouveau visage de la gouvernance des risques en assurance », Revue Banque \& Stratégie, $\mathrm{N}^{\circ} 341,2015$, p.5-8.

FLYNN, K., "Financial fraud in the private health insurance sector in Australia: Perspectives from the industry ", Journal of Financial Crime, Vol. 23, $\mathrm{N}^{\circ}$ 1, 2016, p.143-158.

Gbegi, D. O. ET AdeBisi, J. F., « The new fraud diamond model. How can it help forensic accountants in fraud investigation in Nigeria? ", European Journal of Accounting Auditing and Finance Research, Vol. 1, $\mathrm{N}^{\circ}$ 4, 2013, p.129-138. 
Glaser, B. G. et Strauss, A. L. La découverte de la théorie ancrée, A. Colin, Paris, 2012 [1967].

HoffMAN, D. G. Managing operational risk : 20 firmwide best practice strategies, Wiley, New York, 2002.

Hussenot, A. (2006). Pratiques et usages organisationnels des sciences et technologies de l'information et de la communication. Paper presented at the Colloque International en Sciences de l'Information, Rennes.

I.F.A.C., The auditor's responsibilities relating to fraud in an audit of financial statements - International Standard on Auditing, 2009.

Illich, I. Une Société sans école traduit de l'anglais par Gérard-Henri Durand, Éditions du Seuil, Paris, 1971.

Jebrin, A. H., ABU-Salma, A. J., " Conceptual Knowledge Approach to Operational Risk Management (A Case Study) », International Journal of Business and Management, Vol. 7, № 2, 2012.

JICK, T. D., « Mixing Qualitative and Quantitative Methods: Triangulation in Action », Administrative Science Quarterly, Vol. 24, N 4, 1979, p.602-611.

Jullien, F. L'écart et l'entre leçon inaugurale de la chaire sur l'altérité, 8 décembre 2011, Galilée, Paris, 2012.

Kassem, R. et Higson, A. W., " The new fraud triangle model », Journal of Emerging Trends in Economics and Management Sciences, Vol. 3, $\mathrm{N}^{\circ}$ 3, 2012, p.191-195.

Kemmis, S., McTaggart, R., Participatory Action Research. Communicative Action and the Public Sphere. dans The Sage Handbook of Qualitative Research, Sage Publications, Los Angeles, 2005, p.271330.

LAFFort, E., CARgnello-Charles, E., « Appropriation croisée : vers une diminution du risque de fraude opérationnelle. Application à la finance de marché », Gestion 2000, Vol. 5, N 13, 2013, p.131-143.

LANIER, M. ., " Epidemiological Criminology: Definition and Application », Journal of Theoretical and Philosophical Criminology, Vol. 2, $\mathrm{N}^{\circ} 1$, 2010, p.63-103.

MackeVicius, J. ET GiRIUnAS, L., " Transformational research of the fraud triangle », EKONOMIKA, Vol. 92, $\mathrm{N}^{\circ}$ 4, 2013, p.150-163.

Meric, J., PesqueuX, Y., Sole, A. La "société du risque": analyse et critique, Economica, 2009.

Mikes, A., "Convictions, conventions and the operational risk maze: the cases of three financial services institutions », International Journal of Risk Assessment and Management, Vol. 7, № 8, 2007, p.10271056.

OKURA, M., " The relationship between moral hazard and insurance fraudnull ", The Journal of Risk Finance, Vol. 14, N 2, 2013, p.120-128.

OuANiCHE, M., La fraude en entreprise comment la prévenir, la détecter, la combattre, Paris 2nd édition, Maxima-L. Du Mesnil, 2015

Ouimet, G., « Criminel en col banc de grande envergure. Un renard bien cravaté. », Psychologie française, Vol. 56, No 4, 2012, p.239-258. 
Pathak, J., PAтHAK, J., " Risk management, internal controls and organizational vulnerabilities », Managerial Auditing Journal, Vol. $20, \mathrm{~N}^{\circ} 6,2005$, p.569-577.

PaUl, J., "Between - Method Triangulation in Organizational Diagnosis », The International Journal of Organizational Analysis, Vol. 4, $\mathrm{N}^{\circ} 2$, 1996, p.135-153.

Power, M. K., " The risk management of nothing », Accounting, Organizations and Society, Vol. 34, $\mathrm{N}^{\circ}$ 6-7, 2009, p.849-855.

Rejesus, R. M., Little, B. B. et Lovell, A. C., « Using data mining to detect crop insurance fraud: is there a role for social scientists? », Journal of Financial Crime, Vol. 12, $\mathrm{N}^{\circ}$ 1, 2005, p.24-32.

SAVAll, H., ZARDET, V., BoJe, D. M. Recherche en sciences de gestion approche qualimétrique, observer l'objet complexe, Économica, Paris, 2004.

Smaili, N., Labelle, R., Stolowy, H., « La publication d'une information financière non conforme à la loi et aux normes : déterminants et conséquences ", Comptabilité - Contrôle - Audit, Vol. 15, $\mathrm{N}^{\circ} 1$, 2009, p.159.

Sutherland, E. H., «White-Collar Criminality », American Sociological Review, Vol. 5, $\mathrm{N}^{\circ} 1,1940$, p.1-12.

Sutherland, E. H., " Is "White Collar Crime" Crime? », American Sociological Review, Vol. 10, № 2, 1944, p.132-139.

Sutherland, E. H., Cressey, D. R. et Luckendill, D. F., Principles of Criminology (version Kindle), Oxford, Rowman \& Littlefield, 1992 [1934]

SwABY, G., " Insurance law reform: deterring fraud in the twenty - first century ", International Journal of Law and Management, Vol. 53, $\mathrm{N}^{\circ}$ 6, 2011, p.413-434.

TORre-EnCiso, M. I., BARros, R. H., « Operational Risk Management for Insurers », International Business Research, Vol. 6, $\mathrm{N}^{\circ} 1,2013$, p.1-11.

TSENG , L. M. ET Su, W. P., " Customer orientation, social consensus and insurance salespeople's tolerance of customer insurance frauds », International Journal of Bank Marketing, Vol. 31, $\mathrm{N}^{\circ}$ 1, 2013, p.38-55.

WeICK, K. E., Enactment Processes in Organizations. dans Making Sense of the Organization, Blackwell Publishing, Oxford, UK ; Malden, MA, 2001 [1977], p.179-206.

WeICK, K. E., Enacted Sensemaking in Crisis Situations. dans Making Sense of the Organization, Blackwell Publishing, Oxford, UK ; Malden, MA, 2001 [1988], p.224-240. 